\title{
Adding Value and Facilitating Data Reuse: the Case of the 4TU.Centre for Research Data
}

\author{
Maria Cruz $^{1}$, Egbert Gramsbergen ${ }^{1}$ \\ ${ }^{1} 4$ TU.Centre for Research Data, TU Delft Library, Delft University of Technology, Prometheusplein 1, \\ 2628 ZC Delft, The Netherlands \\ Corresponding author: Maria Cruz (M.J.MarquesdeBarrosCruz@tudelft.nl)
}

\begin{abstract}
The history of the 4TU.Centre for Research Data goes back to 2008, when it started as a project of the libraries of three technical universities in the Netherlands. The aim was to serve the data curation needs of heterogeneous research communities. Fast forward ten years, and over $90 \%$ of the data stored in the 4TU archive are geoscientific datasets coded in netCDF (Network Common Data Form). This is a data format and model that, although generic, is mostly and widely used in atmospheric sciences and oceanography. As an endeavour to ensure that the 4TU.Centre for Research Data remains relevant and successful in the long term, we are exploring options for expanding the services related to netCDF data and potentially build a community of netCDF data depositors and users. Here we present the results of semi-structured, qualitative interviews with eleven researchers, all based in the Netherlands, who use and produce netCDF data; nine of them deposited netCDF data in the 4TU archive. These researchers represent heterogeneous research communities within the Earth sciences, with different views and attitudes to data archiving and publishing. Any new services or community building attempts will need to take this diversity into account. A common need for training and advice may guide the way forward for the 4TU.Centre for Research Data.
\end{abstract}

\section{Introduction}

The 4TU.Centre for Research Data (formerly known as '3TU.DataCentrum') was started in 2008 as a collaboration of the libraries of three universities of technology in the Netherlands: Delft University of Technology (TU Delft), Eindhoven University of Technology, and the University of Twente. The ambition was, and still is, to create and maintain a national state-of-the-art facility for storing and preserving science and engineering research data, and for making those data openly accessible. The 4TU data archive has been fully operational since 2010 and it has evolved to become a trusted and certified repository for science and engineering. As of 30 April 2018, the archive held 7581 datasets, corresponding to about 32.6 TB of data.

The 4TU archive was originally built "as a data curation facility to meet the diverse needs of heterogeneous research communities" (Rombouts \& Princic 2010). Although it contains, and still attracts, heterogeneous data types, $90 \%$ of the data stored in the 4TU archive are environmental research data coded in netCDF (Cruz et al. 2018). Therefore, the 4TU.Centre for Research Data has a special interest in this area and it offers specific services and tools to enhance the access to and the use of netCDF datasets. In recent work (Cruz 2018, Cruz et al. 2018), we argued that repositories need to have a subject or format focus to remain relevant and successful in the long term. In the case of the 4TU.Centre for Research Data, that means exploring our options for providing further services related to netCDF data, be it technical services or training and guidance.

NetCDF is described by its authors as "a set of software libraries and self-describing, machineindependent data formats that support the creation, access, and sharing of array-oriented scientific data" (Unidata 2018). For netCDF datasets, besides the usual download, the 4TU archive offers access via the OPeNDAP (Open-source Project for a Network Data Access Protocol) protocol, the 
main advantage of which is the ability to inspect files (and metadata hidden within the files) and retrieve subsets of files without the need to download whole datasets.

\section{Methods}

To assess what expanded or novel netCDF services the 4TU.Centre for Research Data could potentially provide to its community of data depositors and users, we conducted nine semistructured qualitative interviews with 11 researchers, all based in the Netherlands, who use and produce netCDF data. Most of these researchers deposited netCDF datasets in the 4TU archive; only two of them hadn't done so. We interviewed researchers at all careers stages, namely: four PhD students, one post-doc, one senior scientist, three assistant professors, one associate professor, and one full professor. They were all geoscientists, working in areas ranging from atmospheric sciences and remote sensing, to hydrology, oceanography, and coastal engineering. They were mostly affiliated with technical universities in the Netherlands, especially TU Delft, but some were based at national research facilities and industry.

The interviews, which were not recorded, lasted around 60 minutes each and were conducted between November 2017 and April 2018. The interviewers took notes of key points during the conversation and wrote preliminary, more extended reports in the day or so after the interview. All interviewees were informed that the findings were going to be published, but were assured that they wouldn't be named and that no information would be individually attributed to them.

\section{Results}

\subsection{An overview of netCDF data stored in the 4TU data archive}

It was clear, even before talking with any data depositors or users, that within the scope of netCDF data, which is mainly used in a limited number of geoscience disciplines, the 4TU.Centre for Research Data is still serving heterogeneous research communities, albeit in the Earth sciences.

In terms of volume, most of the netCDF data stored in the 4TU archive originate from one single experiment - the IRCTR Drizzle Radar (IDRA), developed at TU Delft's International Research Centre for Telecommunications and Radar (IRCTR) and installed on top of the Dutch meteorological observatory at Cabauw in the Netherlands (Otto \& Russchenberg, 2014). This project has contributed with 2325 datasets to date, corresponding to about 27 TB of data (Otto et al. 2010). This is a growing time series of datasets, updated every few months, providing detailed observations of the spatial and temporal distribution of rainfall and drizzle around the radar's location.

The remaining 4198 netCDF datasets (as of 30 April 2018), corresponding to a total of about 3.2 TB of data, are either part of much smaller collections (less than $20 \mathrm{~GB}$ in size), or are individual datasets that range in size from about $600 \mathrm{~KB}$ to $136 \mathrm{~GB}$. These datasets can be broadly classified as environmental research data, ranging from river discharge data (Hellebrand 2004) to measurements of aeolian sediment transport (Hoonhout, de Vries and Cohn 2016), and from climate projections (Mezghani, Dobler \& Haugen 2016) to local mean sea level models (Gerkema \& Duran Matute 2017).

\subsection{Main findings from the interviews}

\subsubsection{Use of the $4 T U$ archive}

In addition to the heterogeneous nature of the datasets, the interviews showed that different communities and individual researchers use the 4TU archive in disparate ways. Some projects chose to store only raw data for long-term preservation; processed data for comparative analyses were stored elsewhere. Other projects stored both raw and processed data together with software and scripts used to process the data. The need for long-term preservation was particularly important for researchers dealing with climate data and long-term data series. Although some of the researchers affiliated with these projects mentioned the need and appreciation for processing and visualisation 
services, they didn't feel this should be a priority or a main role for the 4TU archive. In their opinion, archiving of (mostly) raw data for long-term preservation should be the focus of the 4TU archive.

For many of the researchers we interviewed though, while they appreciated the benefits of longterm preservation and of making their data publicly available, their main motivation to use the 4TU archive was to comply with publisher and journal requirements regarding data availability. In these cases, often only processed, output data used to produce the figures in a journal publication were archived. The potential for data reuse and data citation advantage (Piwowar \& Vision 2014) were an important motivation for most of the data depositors.

Overall, the OPeNDAP services offered by the 4TU archive did not seem to have had much influence in the choice of archive for most of the data depositors we interviewed. A minority of the researchers were not aware of OPeNDAP and its functionalities; many knew about OPeNDAP but were just not fully aware that the 4TU archive provided it as a service; others knew about this service but did not consider it important, mostly because their datasets were not big enough for them to care about retrieving data subsets or metadata without the need to download entire datasets.

Many data depositors chose the 4TU archive because it was locally available at TU Delft; the vast majority of netCDF datasets in the 4TU archive originate from TU Delft. Most data depositors chose the 4TU archive after the recommendation of a colleague, supervisor, or data librarian, suggesting that community building efforts may lead to an increase in the use of the 4TU archive.

\subsubsection{Use of netCDF and training}

For the majority of researchers we interviewed, netCDF is the standard data format and model adopted by their communities and it's the primary data format they use and handle. For a few researchers, netCDF was not a standard in their community. In some cases, netCDF was used out of choice because of its self-describing properties and interoperability; in other cases, it was simply because it was the output format of commonly used models or software packages.

During the interviews, we noticed that some researchers, who would have benefit from the use of OPeNDAP and its functionalities, were not aware of its existence. With a few notable exceptions, we also noticed a general lack of awareness of the importance of metadata, which can be included in netCDF files, and a lack of attention or adherence to metadata standards and conventions.

None of the researchers had had formal training on the use or production of netCDF files. Most of them started using netCDF during their $\mathrm{PhD}$ and learned by reading manuals and documentation, through advice from peers and colleagues, and just simply by trial and error. Asked about receiving formal training, there was a general, but not unanimous recognition that there was a need for it, particularly on the research data management aspects of handling netCDF data (e.g. how to include metadata, what metadata to include, conventions and community standards, etc.). Many of the early career researchers we interviewed, mainly PhD students, where enthusiastic about receiving formal and in-depth training. The more senior researchers recognised the need for training, but mostly for PhD students. At their level, they felt that training put too much of a burden on their already busy schedules. That said, because most researchers didn't learn about netCDF in a structured way, they sometimes had gaps in their knowledge. For example, some researchers noted that it took them a while to learn about useful netCDF tools (e.g. Climate Data Operators) and conventions (Climate and Forecast metadata conventions) that were very useful to them and which they wished they had learned about sooner in their careers. In this sense, short training sessions with high-level information about what is possible and available would be welcome even by busy senior researchers.

\section{Conclusions}

The netCDF data depositors and users of the 4TU archive represent heterogeneous research communities within the Earth sciences. They have different views and attitudes to data archiving and publishing, and store wide-ranging types of netCDF datasets in the 4TU archive. Ensuring that any new and current netCDF services continue to be relevant to these communities will require taking their diversity of needs and requirements into account. A need for training and guidance - 
particularly on data management aspects related to documentation, metadata standards and conventions - may be the common thread uniting these communities. This may provide the way forward for the 4TU.Centre for Research Data to build a community of data depositors and users. Ultimately, as noted by Leonelli (2017), well-informed, inclusive, and participatory development of data infrastructures is expected to lead to an increase in the quality and re-usability of research data.

\section{Acknowledgement}

We are extremely grateful to all the researchers who agreed to speak with us for their time and for their invaluable comments and feedback.

\section{References}

Cruz, M J, Böhmer, J K, Gramsbergen, E, Teperek, M, de Smaele, M and Dunning, A 2018 From Passive to Active, From Generic to Focused: How Can an Institutional Data Archive Remain Relevant in a Rapidly Evolving Landscape? OSF Preprints. DOI: http://doi.org/10.17605/OSF.IO/JGRKB

Cruz, M J 2018 How does a data archive remain relevant in a rapidly evolving landscape: the case of the 4TU.Centre for Research Data. Zenodo. DOI: http://doi.org/10.5281/zenodo.1175238

Gerkema, T and Duran Matute, M 2017 Annual mean sea level in the Dutch Wadden Sea 2009-2011. NIOZ Royal Netherlands Institute for Sea Research. Dataset. DOI:

https://doi.org/10.4121/uuid:115ef6c5-8c58-4905-91f5-537985fb3b6f

Hellebrand, H 2004 All data measured by discharge meter in Attert basin. TU Delft. Dataset. https://doi.org/10.4121/uuid:0e38dcc8-d524-4abf-ab59-5c9a38075dc3

Hoonhout, B M, de Vries, S and Cohn, N 2016 Field measurements on aeolian sediment transport at the Sand Motor mega nourishment during the MegaPeX field campaign. TU Delft. Dataset. DOI: https://doi.org/10.4121/uuid:3bc3591b-9d9e-4600-8705-5b7eba6aa3ed

Leonelli, S 2017 Towards the European Open Science Cloud: Five Lessons from the Study of Data Journeys. Zenodo. DOI: http://doi.org/10.5281/zenodo.1043154

Mezghani, A, Dobler, A and Haugen, J H 2016 CHASE-PL Climate Projections: 5-km Gridded Daily Precipitation \& Temperature Dataset (CPLCP-GDPT5). Norwegian Meteorological Institute. Dataset. https://doi.org/10.4121/uuid:e940ec1a-71a0-449e-bbe3-29217f2ba31d

Otto, T and Russchenberg, H W J 2014 High-resolution polarimetric X-band weather radar observations at the Cabauw Experimental Site for Atmospheric Research. Geosci. Data J., 1: 7-12. DOI: $\underline{10.1002 / g d j 3.5}$

Otto, T, Russchenberg, H W J, Reinoso Rondinel, R R, Unal, C M H and Yin, J 2010 IDRA weather radar measurements - all data. TU Delft. Dataset. https://doi.org/10.4121/uuid:5f3bcaa2-a456-4a66a67b-1eec928cae6d

Piwowar, H A and Vision, T J 2013 Data reuse and the open data citation advantage. PeerJ 1:e175. DOI: https://doi.org/10.7717/peerj.175

Rombouts, J and Princic, A 2010 Building a 'data repository' for heterogeneous technical research communities through collaborations. In: International Association of Scientific and Technological University Libraries, 31st Annual Conference. Paper 10.

http://docs.lib.purdue.edu/iatul2010/conf/day2/10

Unidata 2018 NetCDF 4.6.1 Available at https://www.unidata.ucar.edu/software/netcdf/docs/index.html [Last accessed 30 April 2018]. 